\title{
Modelling the Adaptability of Biological Systems
}

\author{
Eugene Ch'ng*
}

School of Computing and Information Technology, University of Wolverhampton, Wolverhampton, UK

\begin{abstract}
There are millions of species of organisms in estimation today, each possessing different preferences or tolerance towards a multitude of factors; these factors include mainly competition between species and environmental conditions. From a computer modelling point of view, these biological systems could be difficult to implement. However, if a unified formula could be found to measure all of the factors against the preferences of each life form, its potentials for modelling open systems that require multiple external input variables could be significant. This article explores a formula and variations of it as applied first to measure sessile systems - vegetation, which requires multiple variables for determining its fitness, and then to a pilot study exploring its use for dynamic vagile systems. Experiments suggest that using the formula and variations of it on vegetation communities yielded distribution patterns similar to those in natural landscapes. Experiments using the formula in the pilot study showed characteristics of emergent behaviours as each dynamic system seeks its own ecological niche. The findings provided evidence that the formula could be extended for modelling a wide variety of open biological systems.
\end{abstract}

Keywords: Biological systems, modelling, tolerance, adaptability, open systems.

\section{INTRODUCTION}

Biology is the scientific study of carbon-based life forms. The modelling of biological systems using theoretical computer science models has many uses; one of the more important and practical reason is that we may better understand their behaviours and perhaps employ the principles discovered within those systems for s olving complex problems (e.g., $[1,2])$. As such, different paradigms such as process algebras, cellular automata, Lindenmayer systems, Petri nets, Boolean functions, P systems, etc., has been used with the aim of providing an understandable, extensible and computable modelling framework while keeping the needed formalisation to perform mathematical analysis [3]. Others (e.g., [46]) model biological systems in order to understand their societal and physical behaviours or for the purpose of simulating their traits $[7,8]$.

The modelling of biological life from studies of natural living organisms requires a systematic collation of information relating to their preferences and behaviours in response to the benefits of, and threats from localised flora and fauna, in conjunction with the parallel impact of environmental change. The procedure of synthesising organisms from this knowledge into computational models of life necessitates the generalisation and distillation of their behaviours into algorithmic rules. For e xample, bird flock [9] is nothing more than three sets of simple rules - separation, alignment and cohesion). Ants $[1,10]$ on the other hand solve problems by following pheromone trails of other ants. The slime mould $[11,12]$ - thousands of distinct single-celled units moving as separate individuals from their comrades, oscillating between being a single celled creature and a swarm coordinated via the individual release of a common substance called acrasin (or cyclic AMP), enabling them to solve problems

\footnotetext{
*Address correspondence to this author at the School of Computing and IT, University of Wolverhampton, Wolverhampton WV1 1SB, UK; E-mail: e.chng@wlv.ac.uk
}

when food a re scarce [13]. While modelling these behavioural rules requires careful observations, a deeper problem that is being neglected is the attempt to discover a unified approach for de termining the collective fitness of an organism based on its preference or tolerance to competition and environmental conditions. Such studies are often lacking. Researchers frequently provide a simple two-state (dead or alive) competition between organisms. For example, Deussen et al.'s [14] simulation of plant competition involves only two states - if two circles intersect, the smaller plant dies, and plants reaching a set size limit are considered old and eliminated. Lane and Prusinkiewicz's method [15] is a little more complex using a probability technique - the parameter $c$ is used for interaction between plants, which sets $c=1$ if the plant is not dominated and to 0 if the plant is dominated. Shade competition replaces the two state domination parameter by $\mathrm{i}$ ntroducing a probability of 1 shaded[sp], where $s p$ is a plant identifier, and shaded[sp] is the shade tolerance of the plant, measured by how likely it is to survive in shadow. Senescence of plants is modelled by introducing a survival probability measure oldage[sp]. This is a far better approach, but does not reflect the interaction of biological systems in the real world.

This article explores a unified method for measuring factors that may affect an organism in its lifespan by taking into account its preference or tolerance towards those factors. These factors may be inter-species competition or critical environmental factors such as temperature, humidity, altitudes, energy from food sources, and liquid that will affect the existence of $a b$ iological system. The present research selects vegetation as the modelling subject as its habitat fulfils the objective for modelling complexity. The article begins with strategies for selecting a target biological system for experiments followed by how biological preferences are defined in the research. Section four investigates the adaptability measure and section five explores how the fitness measure can be applied to a biological system. Section six covers experiments with vegetation adaptability and intro- 


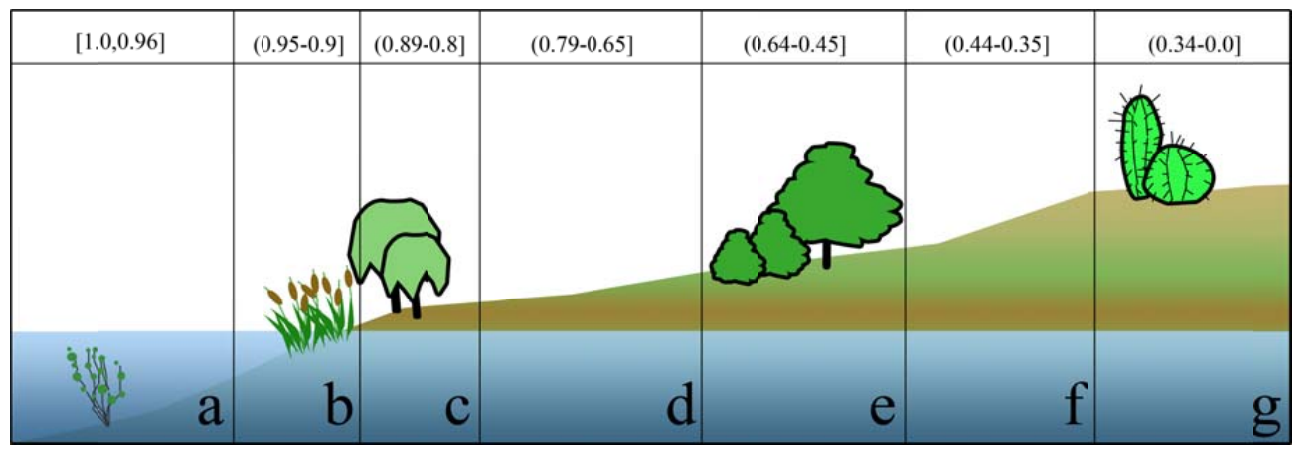

Fig. (1). An example of how vegetation preferences are modelled. The illustration showed plant adaptability towards hydrology and soil moisture content.

duced a pilot study with dynamic systems before concluding the article with a discussion of the findings and potential application areas.

\section{STRATEGIES FOR MODELLING}

Not all biological systems are equal, in order to find a solution to our problem, a particular biological system needs to be identified. The cybernetic system should fulfil certain criteria so that the factors affecting the system can represent all other system. The first criterion is that it must be an open system where matter or energy can flow into and out of the system. The second criterion is that the system should depend on more factors for its survival than alternate systems. The third criterion is that the system necessarily interacts with the three layers of the environment - air, land, water and with other organisms (via competition or symbiosis). The fourth criterion is that the system will expire if one of the layers in the third criterion is removed from the system. Vegetation fulfils all of these criteria.

Vegetation is an open system. It receives matter from the ground and energy from the sun. A typical plant depended on many factors for its survival - water, sunlight, soil, carbon dioxide, etc. as compared to a fish for example which does not require soil for s urvival, or birds, which requires even lesser factors for its existence. Vegetation therefore, is a suitable system for the study.

\section{BIOLOGICAL PREFERENCES}

All biological systems possess unique traits that enable them to survive and thrive in their own ecological niche. These traits can be defined as their preferences (suitability), or negatively as tolerance (level of extremity an organism can tolerate). Different species of ve getation possesses different preferences towards the environment. In fact, preferences are defined as tolerance or adaptability [16]. These preferences can be described using variables - the genetic makeup of the species. The table below shows an example of the genetic makeup of $p$ lant tolerance to extremes of temperatures in the present research.

Table 1. An Example of the Genetic Makeup of Plant Adaptability to Extremes of Temperatures

\begin{tabular}{|c|c|c|c|}
\hline Plant Species & Lower & Preferred & Upper \\
\hline \hline Hot Climate & $-15^{\circ} \mathrm{C}$ & $8^{\circ} \mathrm{C}$ & $25^{\circ} \mathrm{C}$ \\
\hline Temperate & $-7^{\circ} \mathrm{C}$ & $10^{\circ} \mathrm{C}$ & $27^{\circ} \mathrm{C}$ \\
\hline Cold Climate & $-6^{\circ} \mathrm{C}$ & $18^{\circ} \mathrm{C}$ & $35^{\circ} \mathrm{C}$ \\
\hline
\end{tabular}

In the present research, preferences that are well defined in botany is described with standard values whereas those which are not are measured between [0,1]. For example, temperature preferences of each species of plants are well defined. Tolerance to sunlight however, is often vague, with descriptions such as "full sun", "partial shade", "shady areas", and etc. Hydrological preferences are similarly described with a relative comparison between the ecology of interspecies preferences from studies of population concentrations. Grime, Hodgson and Hunt's Comparative Plant Ecology [17] is one example. In the simulation, these plant preferences use a relative measure. Fig. (1) illustrates an example approach used in the research presented here for modelling hydrological preference for plants, other preferences which are vaguely described in related literatures use similar approach.

In band $a$, the preference of aquatic plants is between 1.0 and 0.96 . Band $b$ and $c$ shows the preference of plants that lived near water sources. These could be Cattails, Papyrus, Willows, and etc. Band $d, e$, and $f$ are land based plants with plants in band $e$ having a moderate preference. Plants preferring dry conditions can be seen in band $g$.

\section{BIOLOGICAL ADAPTIBILITY}

Adaptation in vegetation denotes avoidance and tolerance to environmental hazards [16]. For example, four main kinds of adaptation to fire are:

1. Resistance where plants have thick, fire-proof bark,

2. Regeneration by s prouting from root stocks or s urviving stems,

3. Possession of specialised underground organs like the lignotubers of certain Eucalyptus species,

4. Specialised, long-lived fruits which accumulate on the plant over a number of years, only opening to release their seeds after the passage of a fire.

Drought tolerant plants adapt by improving their water relations both by increasing their efficiency in extracting and storing water, and by reducing the rate at which they lose water through evapotranspiration [18]. Many plant species are killed by even the briefest exposure to water-logging. Herbaceous vascular plants from permanently wet sites show a range of morphological and physiological traits including:

1. Anatomical features allowing oxygen transportation to the roots, 


\section{The ability to exclude or tolerate soil toxins, and}

3. Biochemical features which allow prolonged fermentation in the roots [19].

Other species, like Populus deltoids, can even germinate underwater and their seedlings can survive considerable periods of submersion [20]. Plants living in permanent shade include Ferns, mosses and lichens, as well as vascular plants of the undergrowth in evergreen forests. These species must maximise their photosynthesis gain from the low levels of energy they receive, by means of reduced respiration rate, increased unit leaf rate, increased chlorophyll per unit leaf weight, increased leaf area per unit weight invested in shoot biomass $[21,22]$. Plants that adapt to low nutrient availability are generally small in size and have a tendency to have small, leathery, long-lived leaves, and a high root : shoot ratios [23, 24]. Other physiological traits include slow growth rates, efficient nutrient utilisation, efficient mechanisms of internal nutrient recycling to ensure minimal losses through leaf fall, exudation or leaching [25]. Plants found to adapt to extremes of cold temperatures often possess small, long-lived leaves. Carbohydrate storage organs allow them to grow rapidly in the spring and also to accumulate resources over several brief growing seasons before investing in a burst of s eed production. In extremes of hot temperatures, plants show small, dissected leaves which increase the rate of convective heat loss and physiological tolerance of very high tissue temperatures [16]. Adaptations of plants in these studies showed that extreme environmental conditions may be countered by developing traits tolerant of surviving in hazardous settings. The adaptability measure presented here refers to the vegetation's developed adaptable traits, modelling the development of phys iological traits are beyond the scope of this research.

The preferences of plants vary across different species and it is known that certain plants are more tolerant to certain environmental conditions than others. The formula below is an equation that can be applied to measure each competition or environmental factor that a plant senses. Favourable conditions that suit the plant's preference will maintain its fitness whereas harsh conditions may decrease it collectively over time to the eventual termination of the plant life.

An adaptability measure (Equation 1) for m easuring the fitness of a plant based on a single competition or environmental factor is therefore,

$$
A_{i}= \begin{cases}1 & \text { if } p_{i}-b\left|p_{i}-L_{i}\right| \leq C \leq p_{i}+b\left|p_{i}-U_{i}\right| \\ -\frac{1}{\left|U_{i}-p_{i}+b\right| p_{i}-U_{i} \mid}\left|C-p_{i}+b\right| p_{i}-U_{i}||+1 & \text { if } p_{i}+b\left|p_{i}-U_{i}\right|<C \leq U_{i} \\ -\frac{1}{L_{i}-p_{i}-b\left|p_{i}-L_{i}\right|}\left|C-p_{i}-b\right| p_{i}-L_{i}||+1 & \text { if } L_{i} \leq C<p_{i}-b\left|p_{i}-L_{i}\right| \\ 0 & \text { otherwise }\end{cases}
$$

where $C$ is the current environmental condition as a signal from projected temperatures, sunlight, moisture, elevation, and etc., or competition factors (sunlight, space, nutrients), $U_{i}$ and $L_{i}$ are respectively the upper and lower tolerance level; $p_{i}$ is a value denoting an ideal condition for the plant (i.e., its preference); and $b$ is the hardiness of the plant for defining the range extending the ideal condition to its upper and lower tolerance levels. Two other functions are derived from the equation above. The first (Equation 2) m easures only the upper bound tolerance so that any values below the ideal range will yield a full fitness level (1.0). This is useful for situations where the lower bound is not needed. For example, most plants can tolerate open spaces, that is, a plot of land where there is no competition for space from other plants. In this case, the upper bound may represent the amount of space occupied by other plants and are measured. Spaces proceeding below the extended ideal range tolerated by the plant however, are considered as open spaces, which yield a full fitness level for the plant. The second variation (Equation 3) is the exact reverse of the first, measuring only the lower bound and yielding a full fitness for values proceeding beyond the extended ideal range. For example, the second equation can be used for measuring soil depth $[0,1]$ where the earth has no depth/shallow (0.0), medium depth $(0.5)$, to infinitely deep soils (1.0). The upper bound equation is defined below,

$$
A_{i}^{V_{\text {Vpper }}}= \begin{cases}1 & \text { if } 0 \geq C \leq p_{i}+b\left|p_{i}-U_{i}\right| \\ -\frac{1}{\left|U_{i}-p_{i}+b\right| p_{i}-U_{i}||}\left|C-p_{i}+b\right| p_{i}-U_{i}||+1 & \text { if } p_{i}+b\left|p_{i}-U_{i}\right|<C \leq U_{i} \\ 0 & \text { otherwise }\end{cases}
$$

The lower bound equation is defined below.

$$
A_{i}^{\text {Lower }}= \begin{cases}1 & \text { if } p_{i}-b\left|p_{i}-L_{i}\right| \leq C \leq 1 \\ -\frac{1}{L_{i}-p_{i}-b\left|p_{i}-L_{i}\right|}\left|C-p_{i}-b\right| p_{i}-L_{i}||+1 & \text { if } L_{i} \leq C<p_{i}-b\left|p_{i}-L_{i}\right| \\ 0 & \text { otherwise }\end{cases}
$$

\section{MEASURE OF FITNESS}

The fitness measure determines the health of the plant based on their adaptability towards the niches of its biotic and abiotic environment, which are measured through the equation presented earlier. While the adaptability measure remains unchanged, the fitness measure may be different, or, it can be adapted to different biological systems, depending on which weighted factors are more important to an organism. The fitness function defined below operates and controls the sensitivity of individual plant species towards the environment on each time step,

$f_{i}=C_{i} M_{i} S_{i} T_{i} g_{i}^{p H} g_{i}^{\text {depth }} g_{i}^{\text {cond }}\left[E_{i} w_{1}+N_{i} w_{2}+O_{i} w_{3}\right]$

Where $f_{i}$ is the fitness measure for a s pecies where $f_{i} \in[0,1]$ is the result of the adaptability measure which measures each factor: $C_{i}$ is the tolerance based on competition for space, $M_{i}$ is based on the soil moisture content, $S_{i}$ for sunlight, and $T_{i}$ for temperature. $g_{i}^{p H}$ is based on the soil $p H$ level, $g_{i}^{\text {depth }}$ for soil depth, and $g_{i}^{\text {cond }}$ for ground condition. Factors considered more crucial of which plants are more sensitive to are listed before the brackets. Factors considered non-threatening are weighted. $w_{1}$ to $w_{3}$ are weights for controlling the sensitivity of each plant towards the factor where $\sum_{1}^{n} w_{n}=1, n=3 . E_{i}$ is the tolerance based on the location of a plant at its altitude, $N_{i}$ for nutrient, and 
$O_{i}$ is based on the levels of carbon dioxide in the atmosphere.

\section{EXPERIMENTS}

In order to test the adaptability measure, six experimental scenarios were conducted focusing on di fferent environmental parameters related to the adaptability of $\mathrm{pl}$ ants as static systems. Later, a pilot study was conducted to demonstrate the feasibility of the adaptability measure with regards to the modelling of dynamic systems.

\subsection{Static Systems: Plants}

The plant-based experiments were carried out on a simulation environment implemented using $\mathrm{C \#}$ and DirectX as it required appropriate $3 \mathrm{D}$ vis ualisation technologies for observing the effects. In the experimental scenarios plants were represented as different coloured pinheads (red and blue for the two extremes and green for intermediate tolerance). Initial placements of plants on the landscape are random and distributive and plants compete for space, sunlight, and nutrients. Most scenarios (Fig. 3a-d, f) show a timestamp (Tn) of the screen shot where $n$ is a simulation time in years. The yearly temporal scale is used because the simulation takes into account the seasonal cycle a plant goes through for its growth, distribution and reproduction. The hardiness of each plant (Equation 1) is set to $b=0.5$ for all experiments. Except for the differences in mainly landscape related factors associated with the objective of the study, all environmental parameters follow the seasonal climate. The graphs below shows in sequence the annual trends for temperatures, sunlight, humidity and level of carbon dioxide in the simulation.

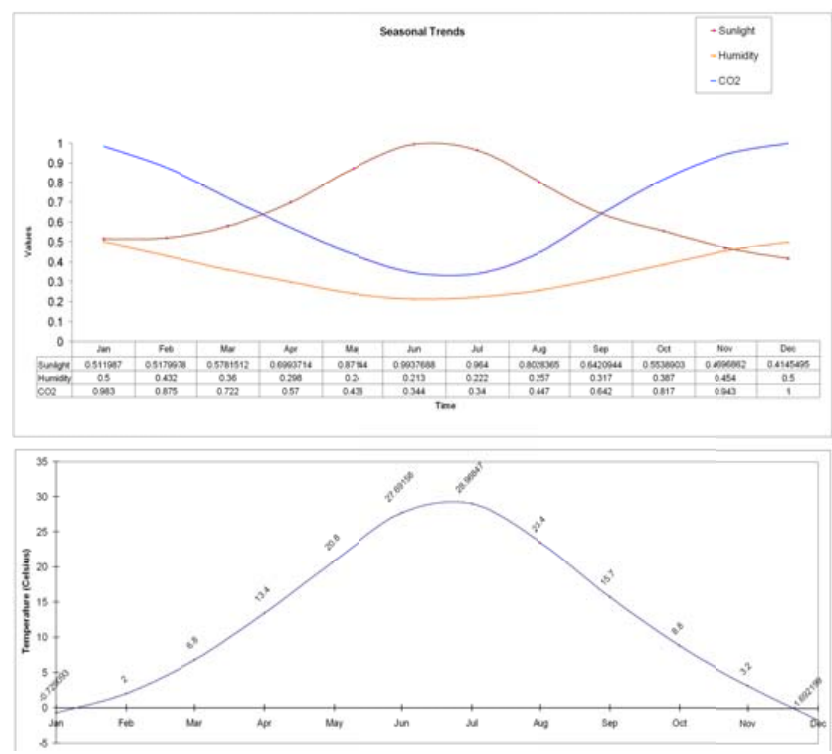

Fig. (2). Graphs showed in sequence the trends for sunlight, humidity, level of carbon dioxide and temperatures in the experiments.

Experiment one focuses on soil depth and its impact on three species of plants. Soil depths are represented as gradients of shallow soils (white regions) to deep soils (black regions) with values ranging from $[0,1]$. Fig. (3a) shows the adaptability of each species of plants on the landscape. Table 2 shows the values used for the adaptability measure for each individual species.

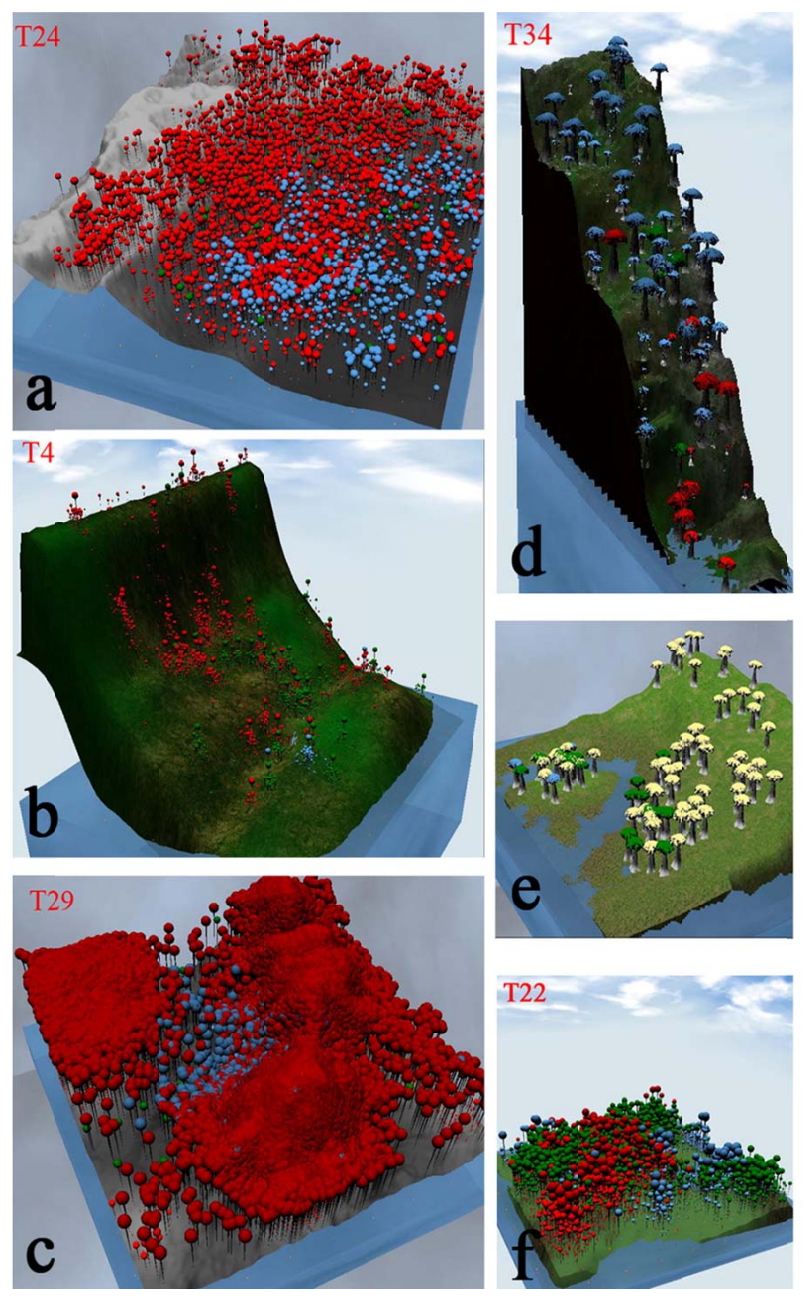

Fig. (3). Experimental scenarios using the adaptability measure: (a) Distribution of species adaptable to different depths of soil. (b) Slope and its effects on di fferent species of vegetation. (c) Soil acidity and its effects on different species of plants. (d) Distribution of plant species in differing temperatures as a result of altitudinal limits. (e) Hydrology and its effects on different species of plants. (f) Layered study of three types of ground condition - soil acidity, soil depth and ground texture.

Table 2. Genetic Makeup of Plant Adaptability to Different Soil Depth

\begin{tabular}{|c|c|c|c|}
\hline Plant Types & Lower & Preferred & Upper \\
\hline \hline Red (Shallow) & 0.09 & 0.2 & 1 \\
\hline Green (Moderate) & 0.3 & 0.5 & 1 \\
\hline Blue (Deep) & 0.6 & 0.8 & 1 \\
\hline
\end{tabular}

Experiment two focuses on the slope condition of a landscape. Fig. (3b) shows the adaptability of each species of plant on the slopes. The population of the red species are seen throughout the landscape, especially on the slopes. Green species populates the middle section of the landscape and the blue species covers small plots of flat lands. Table 3 
shows the values used for the adaptability measure for each individual species.

Table 3. Genetic Makeup of Plant Adaptability to Different Slope Conditions

\begin{tabular}{|c|c|c|c|}
\hline Plant Types & Lower & Preferred & Upper \\
\hline \hline Red (Steep) & $20^{\circ}$ & $70^{\circ}$ & $83^{\circ}$ \\
\hline Green (Moderate) & $20^{\circ}$ & $45^{\circ}$ & $55^{\circ}$ \\
\hline Blue (gentle) & $0^{\circ}$ & $15^{\circ}$ & $35^{\circ}$ \\
\hline
\end{tabular}

Experiment three (Fig. 3c) focuses on soil acidity. Black areas on the landscape contain high levels of alkaline $(\mathrm{pH} 0)$ while white represents areas with the highest level of acidity ( $p H 14)$. Table 4 shows the genetic makeup of each species.

Table 4. Genetic Makeup of Plant Adaptability to Different Soil Acidity

\begin{tabular}{|c|c|c|c|}
\hline Plant Types & Lower & Preferred & Upper \\
\hline \hline Red (High) & $\mathrm{pH} 11$ & $\mathrm{pH} 8$ & $\mathrm{pH} 14$ \\
\hline Green (Moderate) & $\mathrm{pH} 4$ & $\mathrm{pH} 7$ & $\mathrm{pH} 9$ \\
\hline Blue (Low) & $\mathrm{pH} 0$ & $\mathrm{pH} 3$ & $\mathrm{pH} 5$ \\
\hline
\end{tabular}

Experiment four (F ig. 3d) focuses on plant adaptability towards temperature extremes as a r esult of the change of altitude. The height of the landscape is $288.5 \mathrm{~m}$. The temperature-altitude ratio for the study is set to decrease by $-7^{\circ} \mathrm{C}$ over $100 \mathrm{~m}$ in elevation. The blue species is dominant on the middle to upper elevation in this study. Table $\mathbf{5}$ shows the genetic makeup of each species for the experiment.

Table 5. Genetic Makeup of Plant Adaptability to Temperatures

\begin{tabular}{|c|c|c|c|}
\hline Plant Types & Lower & Preferred & Upper \\
\hline \hline Red (Hot Climate) & $-15^{\circ} \mathrm{C}$ & $8^{\circ} \mathrm{C}$ & $25^{\circ} \mathrm{C}$ \\
\hline Green (Temperate) & $-7^{\circ} \mathrm{C}$ & $10^{\circ} \mathrm{C}$ & $27^{\circ} \mathrm{C}$ \\
\hline Blue (Cold Climate) & $-6^{\circ} \mathrm{C}$ & $18^{\circ} \mathrm{C}$ & $35^{\circ} \mathrm{C}$ \\
\hline
\end{tabular}

Experiment five (Fig. 3e) focuses on plant adaptability towards hydrology. It can be observed that the yellow species dominated most of the landscape due to its large range of adaptability. Table 6 shows the genetic makeup of each species for this study.

Table 6. Genetic Makeup of Plant Adaptability to Hydrology

\begin{tabular}{|c|c|c|c|}
\hline Plant Types & Lower & Preferred & Upper \\
\hline \hline Yellow (Intolerant) & 0.02 & 0.28 & 0.67 \\
\hline Green (Intermediate) & 0.13 & 0.43 & 0.82 \\
\hline Blue (Tolerant) & 0.3 & 0.58 & 0.96 \\
\hline
\end{tabular}

Experiment six (Fig. 3f) is a study based on a mixture of different soil conditions. Three different soil types were used for the experiment - soil acidity, soil depth, and soil texture. The results showed a natural distribution of $\mathrm{pl}$ ants in the landscape as a result of the blending of the soil conditions. Table 7 shows the values related to soil texture used for each individual species of plants. Soil depth and soil acidity follows that of Tables $\mathbf{2}$ and $\mathbf{4}$. Table $\mathbf{8}$ shows the plant adaptability for crowded spaces used in all six scenarios.

Table 7. Genetic Makeup of Plant Adaptability to Soil Textures

\begin{tabular}{|c|c|c|c|}
\hline Plant Types & Lower & Preferred & Upper \\
\hline \hline Red Species & 0.3 & 0.8 & 0.95 \\
\hline Green Species & 0.3 & 0.5 & 0.75 \\
\hline Blue Species & 0 & 0.25 & 0.5 \\
\hline
\end{tabular}

Table 8. Genetic Makeup of Plant Adaptability to Crowded Spaces

\begin{tabular}{|c|c|c|c|}
\hline Plant Types & Lower & Preferred & Upper \\
\hline \hline All Species & 0 & 0.35 & 0.5 \\
\hline
\end{tabular}

\subsection{Dynamic Systems}

The pilot experiments conducted here represents an extension of the study for demonstrating that the adaptability measure can also include dynamic systems - biological systems that move.

The study is simple. Two emitters are placed onto a $2 \mathrm{D}$ simulation environment. The red emitter represents a heat source generating $T_{j}=100^{\circ} \mathrm{C}$ of heat from its centre which decreases in temperature using Equation 5 where $d$ is the distance between the emitter and a biological system represented as an agent in the Virtual Environment. The blue emitter represents a cooling source radiating cold temperatures $T_{j}=-100^{\circ} \mathrm{C}$ from its centre using the same equation for the increase in temperature.

$\mu=T_{j}\left(e^{d / T_{j}}\right)^{-1}$

Where $d$ is the distance between the emitter and a biological system represented as an agent in the Virtual Environment. There are three types of agents with three different sets of genetic makeup. The blue species prefers cold temperatures and the red species prefers hot temperatures. The green species is intermediate but leans toward the hot preference type. Each agent is blind but is attracted by its temperature preferences. Each agent senses its environment for temperature sources and its internal system evaluates its fitness via the adaptability measure with a hardiness of $b=0.55$. In this case, the fitness would simply be the value generated by the adaptability measure since its existence requires only the temperature as compared to multiple factors for the plants,

$f_{i}=A_{i}$

Where $i$ is an agent, and $A_{i}$ is the output from the adaptability measure. The simple behavioural rules defined in the heat-seeking agents are as follows: 


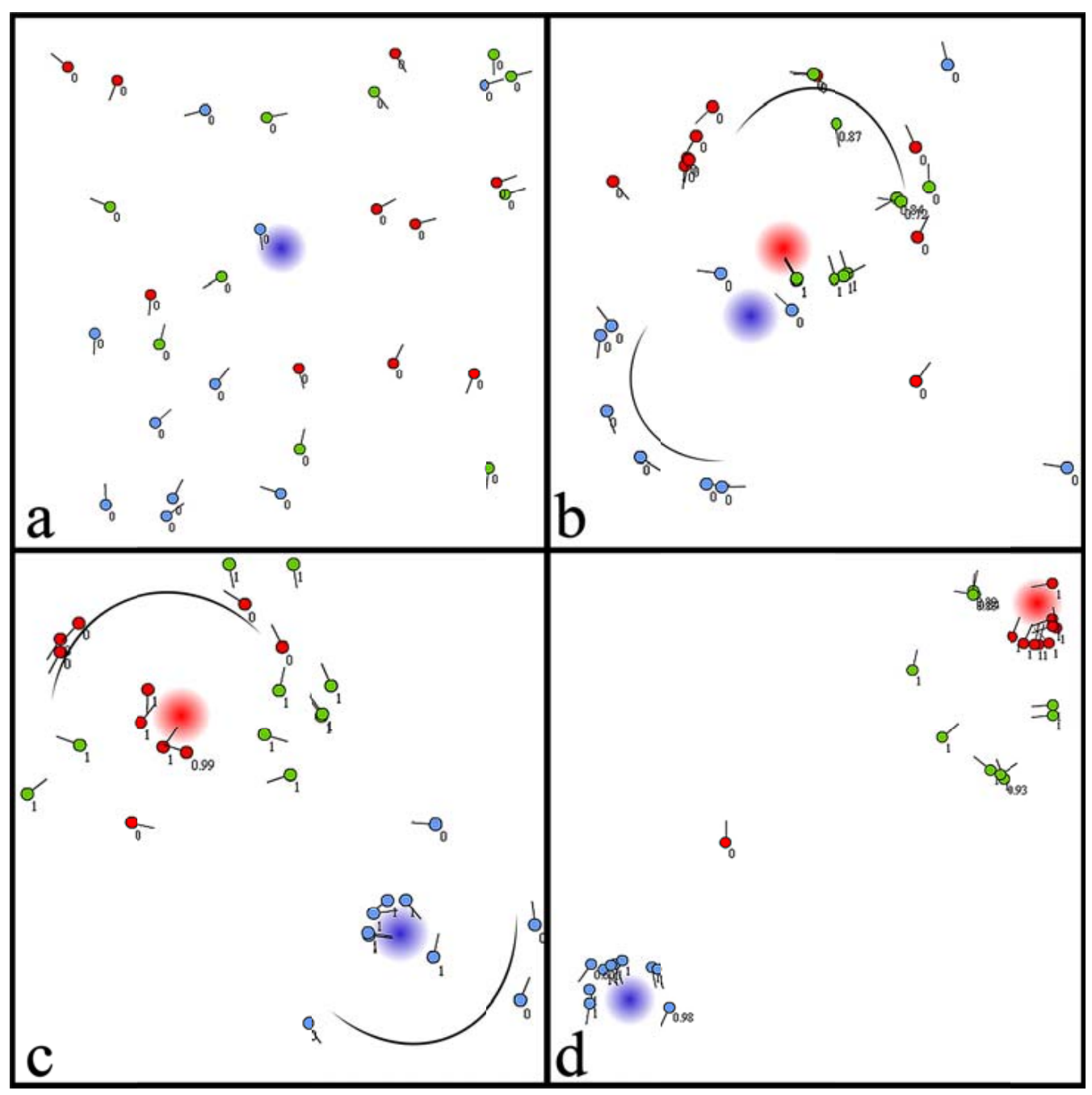

Fig. (4). Experimental scenarios on dynamic systems: (a) Mergence of the heat and cooling emitters. (b) The heat and cooling emitters in close range. (c) The heat and cool emitters in medium range. (d) The heat and cooling emitters in large range. The arrows in (a) and the curve lines (b to c) illustrate the direction of travel.

1. Sense and evaluate the temperature in its immediate surroundings

2. Do not change direction if it is getting hotter. Vice versa for the cold-seeking agent

3. If it is getting colder, change angle of movement. Vice versa for the cold-seeking agent

4. Rest when ecological niche is found

Fig. (4) shows the same scenario with different distances between the emitters. A fitness value reflecting its health accompanies each agent. An agent with a fitness of 1 shows that it is in an adaptable location, this turns the agent's state into the 'rest' mode. A fitness of lesser than 0.8 will keep the agent in a roaming state. Each scenario is allowed to run for 1.5 minutes. In (Fig. 4a), when the emitters are overlapped, the temperatures crossed out each other and the agents roam in alternate directions, having not found its ecological niche, in this case - a s uitable temperature region. The arrows pointing in parallel directions with some agents are added later to illustrate the direction of their movement. When the emitters are moved to a small distance from each other, such as in (Fig. 4b), emergent behaviours are observed. The intermediate agent (green) found its niche whereas the red and blue agents circles its preferential sources (curve lines show their travelling direction). When the emitters are moved to a medium distance from each other, more red and green agents discovered their niches. Green agents are shown resting a little further away from the red agents where the temperatures are ideal. Fig. (4d) shows a scenario where the emitters are furthest apart. In this case, except for a red agent, most agents discovered their niches and switched to the 'rest' mode where the temperature is ideal.

\section{DISCUSSION}

The way different organisms colonise a landscape manifests different characteristic patterns. Such patterns can be observed in abundance in nature, especially vegetation. For example, Ferns cluster together in abundance around damp landscapes and the growth of certain species of Cactus is sparse in dry de serts. In a forest dominated by $\mathrm{P}$ ines, the Pine species are grouped together in sparse distances with an undergrowth of shade tolerant species. Willows appeared to grow near sources of water. It is said that the environment plays an important role in the colony of biological systems. It is also true to say that it is the adaptability of these biological systems that determines their particular habitat. Find- 
ing a formula that can model the adaptability of these systems requires careful observations and experimentations, much like the modelling of the behavioural rules which constitute these systems.

Experiments on both static and dynamic systems suggests that the formula developed in this research can become useful in areas related to the modelling of life, behavioural, and social systems in living entities. In many of these systems, the entities have certain tolerance toward the conditions of its environment. For example, marine organisms in general have full tolerance to submerged environments and zero tolerance on land. In contrast, land-based mammals subject to how long it can hold its breath have zero tolerance under water. An amphibian on the other hand possesses a balanced adaptability for bot $\mathrm{h}$ submerged and land-based environments. The adaptability measure can be used in such cases. In behavioural and social systems for example, certain people have very little tolerance towards certain behaviours whereas others are broad and generous. The adaptability measure can also be used for measuring the level of attraction of a male-female in the mating games of animal species.

\section{REFERENCES}

[1] E. Bonabeau, G. Theraulaz, "Swarm Smarts," Sci Am, vol. 282, pp. $72-79,2000$

[2] N. Forbes, Imitation of Life: How Biology is Inspiring Computing. USA: MIT Press, 2005.

[3] F. Bernardini, M. Gheorghe, F. J. Romero-Campero, and N. Walkinshaw, "A Hybrid Approach to Modelling Biological Systems" in 8th Workshop on Membrane Computing, WMC8Thessaloniki, Greece, The European Molecular Computing Consortium, pp. 140-164. 25-28 June 2007.

[4] C. K. Hemelrijk, "Emergence of Despotic and Egalitarian Societies", pp. 361-369, 1999.

[5] C. K. Hemelrijk and J. Wantia, "Individual Variation by SelfOrganisation," Neuro Biobehav Rev, vol. 29, pp. 125-136, 2005.

[6] A. Huth, C. Wissel, "The analysis of behaviour and the structure of fish schools by means of computer simulations," Comments on Theoretical Biology, vol. 3, pp. 169-201, 1994.

[7] K. Sims, "Evolving 3D Morphology and Behavior by Competition," presented at Artificial Life IV: Proceedings of the Fourth In- ternational Workshop on the Synthesis and Simulation of Living Systems, 1994.

[8] H. Kunz, C. K. Hemelrijk, "Artificial Fish Schools: Collective Effects of School Size, Body Size and Body Form," Artif Life, vol. 9, pp. 237-253, 2003.

[9] C. W. Reynolds, "Flocks, Herds, and Schools: A Distributed Behavioral Model," presented at Computer Graphics, Siggraph '87 Conference Proceedings, 1987.

[10] R. Gordon, Ants at Work: How an Insect Society is Organized. New York: Free Press, 1999.

[11] M. Morse, "Equilibria in Nature," Proc Am Philos Soc, vol. 93, pp. 222-225, 1949 .

[12] L. A. Segel, "Computing An Organism," PNAS, vol. 98, pp. 3639$3640,2001$.

[13] T. Nakagaki, "Maze-solving by an amoe boid organism," Nature, vol. 407, pp. 470, 2000.

[14] O. Deussen, P. Hanrahan, B. Lintermann, R. Mech, M. Pharr, and P. Prusinkiewicz, "Realistic modeling and rendering of plant ecosystems," presented at Proceedings of SIGGRAPH '98 Annual Conference Series 1998, 1998.

[15] B. Lane and P. Prusinkiewicz, "Generating Spatial Distribution for Multilevel Models of Plant Communities," presented at Proceedings of Graphics Interface '02, 2002.

[16] M. J. E. Crawley, Plant Ecology. Oxford: Blackwell Scientific Publications, 1986

[17] J. P. Grime, J. G. Hodgson, and R. Hunt, Comparative Plant Ecology: A functional approach to common British species. London: Unwin Hyman Ltd., 1988.

[18] R. O. Slatyer, Plant-Water Relationships. London: Academic Press, 1967.

[19] A. H. Fitter, Haw, R.K.M., Environmental Physiology of Plants. London: Academic Press, 1981.

[20] J. R. Clark, J. Benforado, Wetlands of Bottomland Hardwood Forests. New York: Elsevier, 1981.

[21] Bjorkman, "Further studies on differentiation of photosynthetic properties in sun and shade ecotypes of Solidago virgaurea.," Physiologia Plantarum, vol. 21, pp. 84-99, 1968.

[22] O. T. Solbrig, "Studies on the population biology of the genus Viola. II. The effect of plant size on fitness in Viola sororia.," Evolution, vol. 35, 1981.

[23] F. S. Chapin, "The mineral nutrition of wild plants.," Ann Rev Ecol Syst, vol. 11, pp. 233-260, 1980.

[24] P. M. Vitousek, "Nutrient cycling and nutrient use efficiency.," Am Nat, vol. 119, pp. 553-572, 1982.

[25] D. T. Clarkson, J. B. Hanson, "The mineral nutrient of higher plants.," Ann Rev Plant Physiol, vol. 31, pp. 239-298, 1980. 